\title{
NOUVELLE
}

\section{Des souris toujours plus humanisées pour de nouvelles applications thérapeutiques}

Jean-Claude Weill, Claude-Agnès Reynaud
Institut Necker-Enfants malades (INEM), Inserm U1151 - CNRS UMR 8253, université Paris Descartes, Sorbonne Paris Cité, faculté de médecine-site Broussais, 14, rue Maria Helena Viera Da Silva, 75993 Paris Cedex 14, France.

claude-agnes.reynaud@inserm.fr

jean-claude.weill@inserm.fr
> Depuis la description de la première souris « humanisée »- une souris immunodéficiente dans laquelle l'injection de cellules souches hématopoïétiques humaines permet l'implantation d'un système immunitaire étranger -, de nombreuses améliorations ont été apportées à ce modèle. Les modèles les plus utilisés sont:

1. La souris BLT (bone marrow, liver, thymus): il s'agit d'une souris NOD/ SCID (souche non obese diabetic, immunodéficiente pour les lignées $T$ et $B$ ) dans laquelle sont greffés du foie et du thymus foetaux humains; quelques semaines plus tard, des cellules souches hématopoïétiques provenant du même foie fœtal sont injectées et reconstituent la moelle osseuse de la souris [1].

2. Les lignées de souris présentant une inactivation de la chaîne commune $\gamma c^{l}$ du récepteur de I'IL(interleukine) 2 $\left(1 / 2 r \gamma^{-/-}\right)$, soit sous fonds génétique $\mathrm{Balb} / \mathrm{c} / \mathrm{Rag}^{-/-}$(BRG), soit sous fonds NOD/SCID (NSG), dans lesquelles sont greffées des cellules souches hématopoïétiques provenant de sang de cordon; l'inactivation du gène $1 / 2 r \gamma$, qui code pour la chaîne $\gamma c$, une des chaînes également du récepteur de l'IL15, inhibe le développement des cellules natural killer (NK) murines et réduit le rejet des cellules humaines. Ces deux modèles ont été décrits dans un article récent de médecine/sciences [2]. La souris BLT permet le développement le plus complet du système immunitaire humain, quoique le compartiment $B$ soit peu

${ }^{1}$ La chaine $\gamma_{c}(\gamma$ commune) est partagée par les récepteurs de six cytokines : les interleukines IL -2, 4, 7, 9, 15, 21. C'est la chaîne $\alpha$ de ces récepteurs qui confère la spécificité d'action de la cytokine. décrit et semble, comme pour les autres modèles, largement immature ([1] et $S$. Weller, J.-C. W. et C.-A. R, données non publiées).

\section{Une nouvelle lignée de souris optimisée pour le développement des lignées myéloïdes et monocytaires/ macrophagiques}

Dans ces différents modèles, les cytokines permettant le développement du système immunitaire sont d'origine murine, ce qui ne permet pas une différenciation de la lignée myéloïde et des cellules NK humaines. Afin de pallier ce problème, l'équipe de $R$. Flavell a construit une nouvelle lignée de souris $\operatorname{Rag2}^{-/-} / 12 r \gamma^{/-}$ dans laquelle les gènes codant pour les cytokines humaines M-CSF (macrophage colony-stimulating factor), IL3, GM-CSF (granulocyte macrophage colony-stimulating factor) et TPO (thrombopoïétine) sont insérés à la place de leur équivalent murin par knock-in (lignée MITRG) [3]. À cette lignée peut être ajouté un transgène codant pour le récepteur SIRP $\alpha$ (signal regulatory protein $\alpha$ ) qui, en se liant au récepteur CD47 présent à la surface des cellules hématopoïétiques humaines, empêche leur phagocytose par les macrophages murins (lignée MI(S) TRG) (Figure 1). Ces souris sont greffées avec des cellules souches $\mathrm{CD}_{3} 4^{+}$provenant du foie fœtal, du sang de cordon ou du sang périphérique adulte. Les souris humanisées développent une myélopoïèse très supérieure à celle qui est décrite dans les modèles précédents; elle inclut des monocytes, des basophiles, des éosinophiles, des cellules dendritiques et des cellules NK humaines, détectées dans le sang et dans les tissus lymphoïdes et non lymphoïdes. Les défauts de ce modèle en ce qui concerne l'immunité adaptative sont semblables à ceux des souris NSG ou BRG: le développement B est immature et la réponse humorale faible au niveau de la commutation isotypique et de l'hypermutation somatique. De plus, le développement des macrophages humains induit une phagocytose par ces derniers des globules rouges de souris, qui entraîne une anémie létale à court terme (2 à 3 semaines). La survie peut être améliorée si l'on n'irradie pas la souris avant la greffe. Les avantages de ce modèle restent notables car il permet d'étudier le rôle des monocytes et des macrophages dans les infections virales ou bactériennes (infection par le VIH [virus immunodéficience humaine], tuberculose), ainsi que le rôle des cellules NK envers les tumeurs. Les propriétés immunosuppressives et anti-inflammatoires des monocytes et des macrophages permettant la réparation tissulaire sont à double tranchant dans les cancers, car elles peuvent favoriser la croissance tumorale ${ }^{2}$ [6]. Une telle situation est démontrée par les auteurs qui, après une xénotransplantation d'un mélanome dans ces souris, observent l'infiltration de macrophages humains avec une polarisation de type $M 2$ secrétant du VEGF et favorisant la croissance de la tumeur $[6,7]$, un processus que seul ce nouveau modèle murin permet d'étudier.

${ }^{2}$ Voir le numéro de médecine/sciences «Microenvironnements tumoraux : contradictoires et complémentaires », publié en avril 2014 (m/s 2014, vol. 30, $\left.n^{\circ} 4\right)$. 


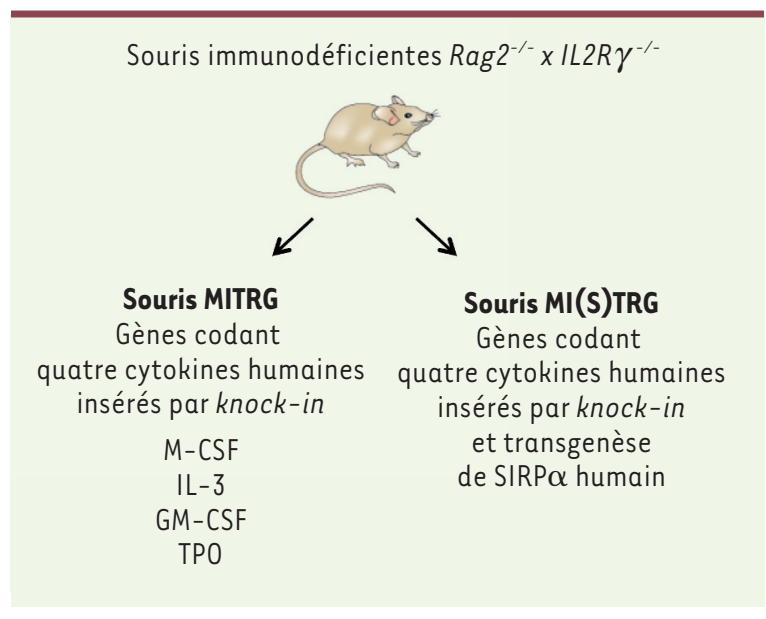

Figure 1. Génération des souris MITRG et MI(S)TRG (d'après [3]).

\section{Production d'anticorps humains} dans une souris contenant la totalité du locus codant pour les gènes variables des Ig humaines

Dans une optique différente, l'humanisation des souris a également porté sur l'insertion de gènes d'immunoglobulines (Ig), afin de produire des immunoglobulines humaines à usage diagnostic et thérapeutique. Une première génération de souris contenant des segments des locus d'Ig humains, comprenant gènes $V$ et $C$, a été construite. Un certain nombre d'anticorps monoclonaux ont été produits par ces souris (sept sont approuvés par la $F D A$ ) [8], mais la présence de segments des régions constantes humaines ne permettait pas une signalisation optimale du BCR ( $B$ cell receptor). De plus, la délétion des locus d'Ig de la souris entraînait une instabilité génétique et un défaut de fertilité du fait de la perte de gènes imbriqués dans ces locus (notamment Adam6a et $6 b$ [a disintegrin and metallopeptidase domain 6B] qui sont curieusement localisés dans la région située entre les gènes $V H$ et $D$, excisée lors du réarrangement des gènes $\left.d^{\prime} \mid g\right)$. Une deuxième génération de souris a été produite par des sociétés de biotechnologie qui, pour la plupart, n'ont pas décrit leurs constructions dans la littérature et contrôlent l'accès à leur modèle.

Le groupe de Allan Bradley (Kymab Ltd, Babraham Research Camus, Royaume-
Uni) vient de décrire, dans le même numéro de Nature Biotechnology où paraît l'article de R. Flavell, un modèle de nouvelle génération dans lequel la totalité du locus codant pour les régions variables des Ig humaines a été insérée dans le locus murin correspondant, en utilisant une technique de recombinaison séquentielle dans des cellules ES (embryonic stem cells) murines [4]. Les segments constants des Ig de souris sont maintenus en place pour préserver la fonctionnalité de l'association du BCR avec les molécules Ig $\alpha$ et $\lg \beta$. Afin d'éviter la délétion de gènes essentiels, les locus $V H$ et $V \kappa$ sont maintenus mais leur utilisation est minimisée par une inversion les relocalisant à quelques millions de bases du locus humain (Figure 2). On reste impressionné par les prouesses techniques déployées. Après avoir inséré une « aire d'atterrissage » (landing pad), quinze modifications successives sont réalisées sur le locus tout en préservant la capacité des cellules ES à repeupler la lignée germinale. Des transposons piggyBac permettent l'auto-excision de I'ADN étranger non humain et donc I'utilisation successive des mêmes marqueurs de sélection. Deux types de souris sont générées, les chaînes légères, assemblées dans des cellules ES différentes, étant apportées par croisement entre lignées de souris: une lignée porte les gènes VH/ $V_{\kappa}$ humains en présence d'un locus lambda murin fonctionnel, et une deuxième les gènes $\mathrm{VH} / \mathrm{V} \lambda$ humains en présence d'un locus kappa murin inactivé (Figure 2). Ces souris produisent des anticorps de différentes classes, ayant subi des mutations somatiques dans les gènes codant pour les régions
$V$ et présentant des CDR3 (complementarity determining regions) ${ }^{3} \mathrm{de}$ longueur semblable à ceux produits chez l'homme (8 à 24 acides aminés). Le développement lymphocytaire $B$ n'est pas décrit dans cette publication. La production de CDR3 de grande taille semble jouer un rôle très important dans la génération d'anticorps neutralisants contre certains épitopes viraux (Influenza, VIH). Dans le cas du $\mathrm{VIH}$, ces épitopes sont enfouis dans des protéines glycosylées qui ne sont accessibles que par des CDR3 exceptionnellement longs. Cette lignée de souris semble donc le modèle idéal pour aborder, par exemple, l'enjeu actuel qui consiste à définir le ou les immunogènes successifs permettant d'obtenir par vaccination des anticorps anti-VIH neutralisants. Les problèmes que pose cette démarche restent néanmoins complexes depuis que l'on sait que ces anticorps avec des CDR3 longs sont souvent autoréactifs et induisent l'élimination des cellules qui les expriment dès leur apparition dans la moelle osseuse. Ceci pourrait expliquer le fait qu'il faut plusieurs années et de nombreuses mutations somatiques pour les faire émerger à partir d'anticorps à très faible affinité et non auto-réactifs lorsqu'ils ne sont pas mutés [5]. II faut souligner qu'un atout majeur de la production d'anticorps humains par transgenèse est la pression de sélection s'exerçant in vivo pour la stabilité et la non-agrégation de ces molécules, probablement responsable de leur faible immunogénicité en usage clinique ${ }^{4}$.

\footnotetext{
3 «La zone de l'anticorps interagissant avec l'épitope est appelée paratope et est localisée au niveau des domaines VH et VL. Six régions, dites hypervariables, localisées au sein de ces domaines variables et appelées CDR (complementarity determining regions), constituent la zone de contact avec l'épitope et confèrent donc la spécificité de liaison à l'antigène » (extrait de [9]).

${ }^{4}$ Voir le numéro de médecine/sciences, «Anticorps monoclonaux en thérapeutique », publié en décembre $2009(\mathrm{~m} / \mathrm{s}$ 2009 , vol. $25, n^{\circ} 12$ )
} 


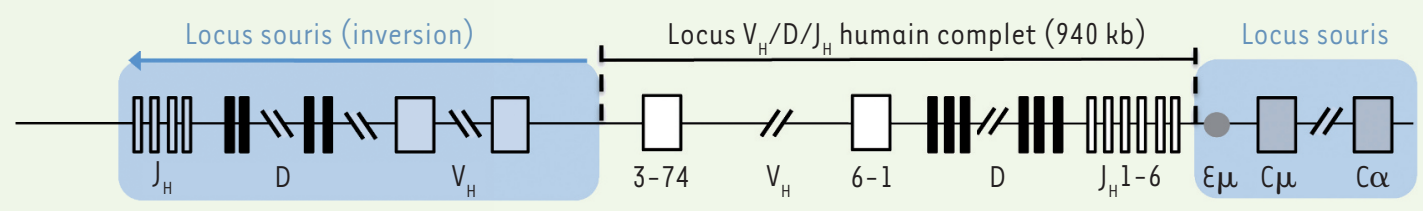

Locus de la chaîne légère kappa

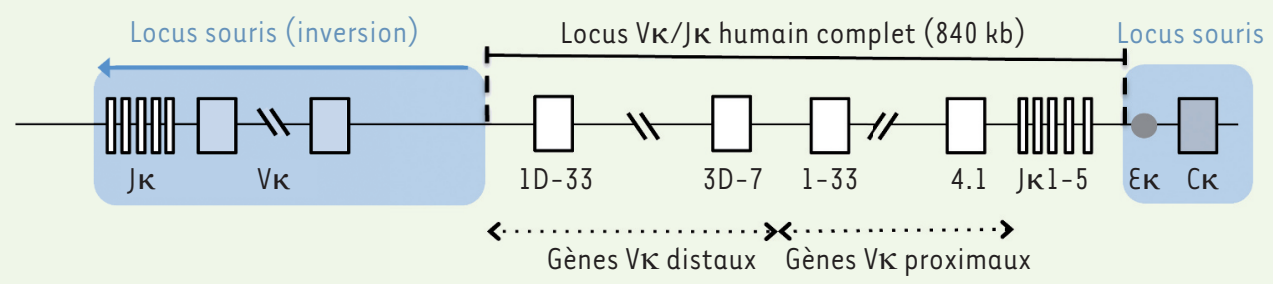

Locus de la chaîne légère lambda

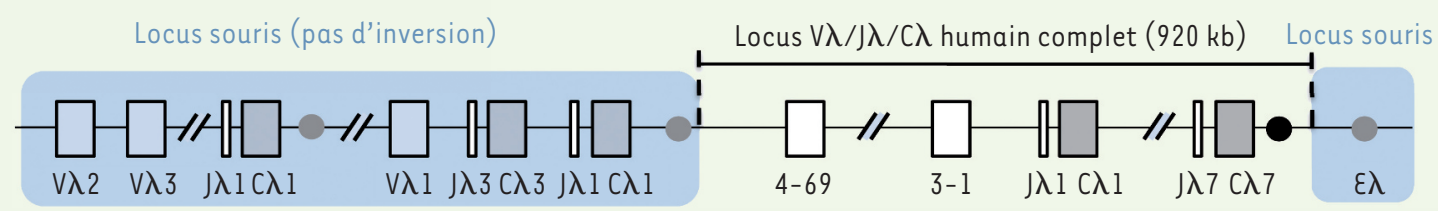

Figure 2. Humanisation complète des loci d'immunoglobulines de souris. L'insertion de la totalité des gènes VH, Vא et V $\lambda$ humains a été effectuée en conservant les enhancers (symboles ronds) et les gènes codant pour les régions constantes de souris, sauf pour le locus de la chaîne légère lambda, où le groupe des 7 gènes $J \lambda$ - $C \lambda$ humains a été également introduit. L'ensemble des gènes variables murins des loci de la chaîne lourde et de la chaîne légère kappa ont été éloignés du locus endogène par une inversion de plusieurs mégabases, rendant ainsi leur utilisation négligeable. Pour le locus lambda qui a été conservé, le petit nombre de gènes $V$ de souris ne semble pas exercer une compétition importante par rapport au locus humain. Le locus VK humain est constitué de deux larges segments palindromiques, qui ont été dissociés et réinsérés en tandem (gènes $V_{\kappa}$ proximaux et distaux) (d'après [4]).

\section{Conclusion}

Ces deux modèles de souris vont permettre d'aborder des questions nouvelles, tant du point de vue fondamental que pour des applications thérapeutiques. Ils devraient de plus être mis à la disposition de la communauté scientifique, notamment pour un usage académique. II reste à comprendre pourquoi les différentes sous-populations $B$ ne se développent pas normalement dans ces modèles d'humanisation par restauration de cellules souches hématopoïétiques humaines. Cela souligne à quel point, contrairement au compartiment $T$, le compartiment $B$ humain semble être différent de celui de la souris. Une meilleure compréhension du développement B chez l'homme devrait permettre sans doute de le reproduire un jour dans une souris humanisée. $\diamond$ Ever more humanized mice for new therapeutic applications

\section{LIENS D'INTÉREิT}

Les auteurs déclarent n'avoir aucun lien d'intérêt concernant les données publiées dans cet article.

\section{RéFÉRENCES}

1. Melkus MW, Estes JD, Padgett-Thomas A, et al. Humanized mice mount specific adaptive and innate immune responses to EBV and TSST-1. Nat Med 2006 ; 12 : 1316-22.

2. Cachat A, Villaudy J, Rigal D, et al. Les souris ne sont pas des hommes et pourtant... Ce que les souris humanisées nous apprennent sur les maladies infectieuses Med Sci (Paris) 2012 ; 28 : 63-8.

3. Rongvaux A, Willinger T, Martinek J, et al. Development and function of human innate immune cells in a humanized mouse model. Nat Biotechnol 2014 ; 32 : 364-72.

4. Lee $\varepsilon C$, Liang $\mathrm{Q}$, Ali $\mathrm{H}$, et al. Complete humanization of the mouse immunoglobulin loci enables efficient therapeutic antibody discovery. Nat Biotechnol 2014 ; 32: 356-63.

5. Verkoczy L, Diaz M. Autoreactivity in HIV-I broadly neutralizing antibodies: implications for their function and induction by vaccination. Curr Opin HIV AIDS $2014 ; 9: 224-34$

6. Bruchard M, Ghiringhelli F. Microenvironnement tumoral : cellules régulatrices et cytokines immunosuppressives. Med Sci (Paris) 2014 ; 30 : 429-35.

7. Provot S. Contrôle de la croissance et de la dissémination tumorales par le microenvironnement : certitudes et hypothèses émergentes. Med Sci (Paris) 2014 ; 30 : 366-71.

8. Cogné $M$, Duchez $S$, Pascal V. Transgenèse animale et humanisation des anticorps. Med Sci (Paris) 2009; 25 : 1149-54.

9. Abès R, Dutertre CA, Teillaud JL. Les anticorps : mieux les connaître pour mieux s'en servir. Med Sci (Paris) $2009 ; 25$ : 1011-9. 